\title{
Combined-spinal-epidural anesthesia in a parturient with gestational hypertension with acute and extremely elevated blood pressure undergoing emergency caesarean section
}

\begin{abstract}
Hypertensive disorder is the most common medical problem encountered in pregnancy. It remains an important cause of maternal, and foetal, morbidity and mortality. Neuraxial anaesthesia techniques are most widely used for caesarean delivery. We describe the anaesthetic management of a 40-year-female with gestational hypertension undergoing caesarean section who presented with acute and extreme rise of blood pressure (BP) $(249 / 116 \mathrm{mmHg})$. Her BP was controlled quickly and she was successfully managed by combined-spinal-epidural technique. This report shows that carefully conducted neuraxial anaesthesia can be useful for caesarean delivery in parturients with severe elevation of BP where general anaesthesia is normally indicated.
\end{abstract}

Keywords: Hypertension; Pregnancy-Induced, Anaesthesia, Obstetrical, Pre-Eclampsia
Volume I Issue 5 - 2014

\author{
Swathi Kumari K, HariharV Hegde \\ Department of Anesthesiology, SDM College of Medical \\ Sciences and Hospital, India
}

\begin{abstract}
Correspondence: HariharV Hegde, Department of Anesthesiology, SDM College of Medical Sciences and Hospital, Dharwad, Karnataka, India, Tel +91836-2477755, Fax +91836246I65I,Email drharryhegde@yahoo.co.in
\end{abstract}

Received: October 19,2014 | Published: November 13, 2014
Abbervations: NHBPEP, National High Blood Pressure Education Program; SAB, Subarachnoid Block; CS, Caesarean Section; GA, General Anaesthesia; EA, Epidural Anaesthesia; CSE, Combined-Spinal-Epidural; RANZCOG, Royal Australian and New Zealand College of Obstetricians and Gynaecologists

\section{Introduction}

Hypertension accounts for approximately a quarter of all antenatal admissions and is the most common medical problem encountered in pregnancy. It complicates up to $15 \%$ of pregnancies and remains an important cause of maternal, and foetal, morbidity and mortality. Hypertensive disorder during pregnancy can exist in four distinct forms as recommended by the National High Blood Pressure Education Program (NHBPEP) working group11: preeclampsia-eclampsia, chronic hypertension of any cause, preeclampsia superimposed on chronic hypertension, and gestational hypertension. ${ }^{2}$ Parturients who experience hypertension in first pregnancy are at an increased risk in a subsequent pregnancy. Risk of recurrence remains greater with earlier onset of hypertension in the first pregnancy. ${ }^{1}$ The definitive treatment being delivery of the foetus and placenta. ${ }^{3}$

Currently, the most widely used anaesthetic technique for caesarean section is neuraxial anaesthesia ${ }^{4}$ For patients with severe preeclampsia without features of impending eclampsia, single shot subarachnoid block (SAB) may be considered a good choice for caesarean section (CS) when compared to general anaesthesia (GA) or epidural anaesthesia (EA). ${ }^{3}$ We describe the anaesthetic management of a 40-year female with gestational hypertension undergoing caesarean section who presented with acute and extreme rise of blood pressure (BP). She was successfully managed by combined-spinalepidural (CSE) technique.

\section{Case presentation}

A 40-year female, weighing $60 \mathrm{~kg}$, gravida 5, abortion 4 and no living child, with 37 weeks gestational age was admitted because of her bad obstetric history to the Department of Obstetrics and Gynaecology of our hospital. She was diagnosed to have gestational hypertension during her fourth pregnancy and gestational diabetes mellitus during the current. She was receiving oral Nifedipine retard $10 \mathrm{mg}$ since 28 weeks of gestation. Her blood sugars were well controlled on diet.

On admission, her heart rate (HR) was 80/min and BP 140/90 $\mathrm{mmHg}$. The dose of oral Nifedipine retard $10 \mathrm{mg}$ was increased to twice daily and later, to thrice daily. Investigations were as follows: hemoglobin $12.2 \mathrm{~g} / \mathrm{dl}$, blood group ' $\mathrm{O}$ ' positive, blood urea $13 \mathrm{mg} / \mathrm{dl}$, serum creatinine $0.6 \mathrm{mg} / \mathrm{dl}$, uric acid $3 \mathrm{mg} / \mathrm{dl}$, platelet count $163000 /$ $\mathrm{mm} 3$, aspartate aminotransferase $14 \mathrm{U} / \mathrm{L}$, alanine aminotransferase 15 $\mathrm{U} / \mathrm{L}$, lactate dehydrogenase- $347 \mathrm{U} / \mathrm{L}$, prothrombine time 12.7 seconds (test) and 12.2 seconds (control), activated partial thromboplastin time 29 seconds (test) and 28 seconds (control), and INR 1.04. Urine routine was within normal limits and there was no proteinuria.

She was scheduled for an elective CS a week later. Two days after the admission, her BP remained elevated $(170 / 100 \mathrm{mmHg})$ in spite of medications. However, there was no history of headache, blurring of vision, epigastric pain, decreased urine output or any other symptoms and signs of preeclampsia. Therefore, she was posted for an emergency caesarean section. In the operating room, her HR was 94/min and non-invasive BP 213/124 mmHg. An arterial line was secured in the right radial artery which showed a BP of 249/116 mmHg (Figure 1A). Intravenous (IV) Labetalol $25 \mathrm{mg}$ ( $5 \mathrm{mg}$ bolus every five min) was administered and the BP was reduced to $189 / 103 \mathrm{mmHg}$ over $20 \mathrm{~min}$.

CSE anesthesia was planned. Under aseptic precautions, epidural space was located at L2-3 space using an 18 G Tuohy needle by loss of resistance to normal saline technique in a single attempt by midline approach in left lateral position. The epidural catheter was fixed at 9 $\mathrm{cm}$ at skin leaving $3 \mathrm{~cm}$ inside the epidural space. Subarachnoid space was located with a $26 \mathrm{G}$ Quicken needle at the same space by left paramedian approach. SAB was achieved with $1.6 \mathrm{ml}$ (including the needle dead space being approximately $0.15 \mathrm{ml}$ ) of $0.5 \%$ hyperbaric 
Bupivacaine. She was then put in supine position with $30^{\circ}$ right lateral tilt. Lower segment caesarean section with a Pfannenstiel incision was commenced. Five minutes after initiating the SAB, her BP decreased to $97 / 56 \mathrm{mmHg}$ and $\mathrm{HR} 68 / \mathrm{min}$. Intravenous Ephedrine $12 \mathrm{mg}$ was administered. The BP increased to $120 / 68 \mathrm{mmHg}$ within the next 90 seconds. Twelve minutes after SAB, a live female baby weighing 2.2 $\mathrm{kg}$ was extracted with an APGAR score of 7 and 8 at 1 and 5 minutes, respectively. Intravenous Oxytocin 2 International Units (IU) bolus was administered followed by infusion of $10 \mathrm{IU}$ in $500 \mathrm{ml}$ normal saline at a rate of $125 \mathrm{ml} / \mathrm{h}$. Soon after extraction of the baby, the BP again decreased to $80 / 40 \mathrm{mmHg}$ which was treated with another bolus of IV Ephedrine $12 \mathrm{mg}$. She received intramuscular Carboprost Tromethamine (15-methyl-PGF2 $\alpha$ ) $125 \mu \mathrm{g}$ as the uterus had not retracted satisfactorily, and IV Pethidine $25 \mathrm{mg}$ intraoperatively for control of shivering.

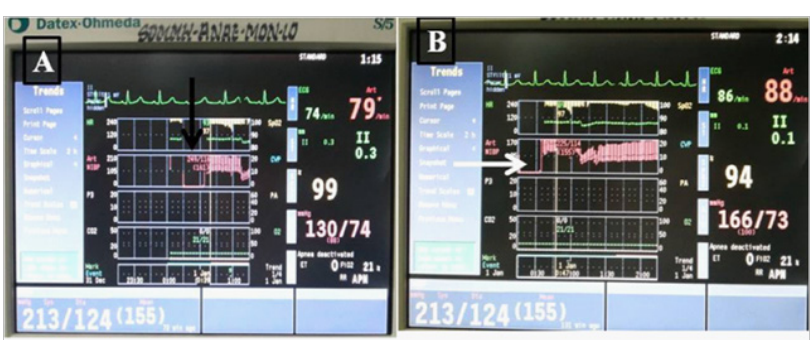

Figure I Trends of vital parameters showing. A) a very high initial blood pressure (arrow). B) transient reduction following induction of anaesthesia, and stabilisation subsequently (arrow).

A total of $2000 \mathrm{ml}$ crystalloids were administered intra operatively. Half an hour after the SAB, her BP showed a rising trend and it was $170 / 60 \mathrm{mmHg}$ towards the end of surgery (Figure 1B). A loading dose of Magnesium sulphate $4 \mathrm{~g}$ in $100 \mathrm{ml}$ normal saline was administered over half an hour followed by an infusion at $1 \mathrm{~g} / \mathrm{h}$ for 24 hours. The duration of surgery was 40 minutes. Epidural analgesia was initiated with $10 \mathrm{ml}$ of bupivacaine $0.125 \%$ with fentanyl $2 \mu \mathrm{g} / \mathrm{ml}$ administered in two aliquots of $5 \mathrm{ml}$ over five minutes with careful monitoring of the haemo dynamics. Total blood loss was estimated to be $600 \mathrm{ml}$. Analgesia was maintained further with infusion of the same at 6 $\mathrm{ml} / \mathrm{h}$. She was shifted to the surgical intensive care unit (SICU) for monitoring.

In the SICU, her HR was $75-85 / \mathrm{min}$, BP $130-170 / 70-90 \mathrm{mmHg}$, and urine output $500 \mathrm{ml}$ over the next 6 hours. After about 12 hours in the SICU, she was transferred to the ward, and epidural analgesia was continued till $24 \mathrm{~h}$ post-operatively. Her BP was well controlled by the $2^{\text {nd }}$ post-operative day. She was discharged a week later. The patient has given consent to publish this report.

\section{Discussion}

\section{Hypertensive disorders of pregnancy can be defined as follows}

Gestational hypertension - is defined as a systolic blood pressure of at least $140 \mathrm{mmHg}$ and/or a diastolic blood pressure of at least 90 $\mathrm{mmHg}$ on at least two occasions after the $20^{\text {th }}$ week of gestation that are a minimum of 4 hours apart (It is considered severe if there are sustained elevations to at least $160 \mathrm{mmHg}$ in systolic blood pressure and $110 \mathrm{mmHg}$ in diastolic blood pressure for at least 4 hours). ${ }^{5}$

Pre-eclampsia - is defined as the new onset of hypertension (blood pressure $>140 / 90 \mathrm{mmHg}$ ) and proteinuria $(>300 \mathrm{mg} / 24 \mathrm{~h})$ after 20 weeks of gestation. Severe pre-eclampsia is the presence of new-onset hypertension and proteinuria accompanied by central nervous system dysfunction (headaches, blurred vision, seizures, coma), marked elevations of BP $(>160 / 110 \mathrm{mmHg})$, severe proteinuria $(>5 \mathrm{~g} / 24 \mathrm{~h})$, oliguria or renal failure, pulmonary oedema, hepatocellular injury (ALT $>2 \mathrm{x}$ the upper limits of normal), thrombocytopenia (platelet count $<100,000 / \mathrm{L}$ ), or disseminated intravascular coagulation.

Eclampsia is defined as the occurrence of a grand mal seizure in association with pre-eclampsia.

\section{Symptoms and signs of severe pre-eclampsia include}

i. Left upper quadrant/epigastric pain due to liver edema $+/$ - hepatic haemorrhage

ii. Headache +/- visual disturbance (cerebral oedema)

\section{iii. Occipital lobe blindness}

iv. Hyper reflexia $+/$ - clonus

\section{v. Convulsions (cerebral oedema).}

The choice of anaesthesia for CS depends on a variety of factors which includes the urgency of the situation; the parturients wishes, and the health of the mother and foetus. Though GA is considered to be the quickest method of anaesthesia in an emergency, the significant drawbacks associated include difficult airway, risk of failed intubation, hypertensive response to laryngoscopy and intubation, risk of aspiration pneumonitis, foetal exposure to depressant medication, an unconscious mother who is unable to participate in the process of birth or interact with the baby once it is delivered. Also, postoperative analgesia is well achieved with regional anaesthesia techniques when compared with GA.

Currently, neuraxial anaesthesia techniques are most widely used for CS delivery and can be used in situations such as severe preeclampsia, and placenta previa where GA is indicated. It is preferred when considering variables such as post-operative pain relief, patient satisfaction and cost effectiveness. ${ }^{4}$ The mother can experience child birth, see and interact with the baby, and start breast feeding early. The safety of spinal and CSE in pre-eclamptic patients for LSCS with no adverse effects on mother or foetus have been demonstrated in multiple studies. ${ }^{6}$

The advantage of the CSE technique is the rapid onset and density of SAB and versatility of EA. If need be, the epidural catheter helps to maintain anaesthesia for prolonged surgery and provide excellent postoperative analgesia as compared with parenteral opioids. It can attenuate the neuro humoral stress response to surgery leading to reduction in maternal catecholamine's, decrease incidence of postoperative ileus, provide effective pain relief without appreciable motor block and improve cardio respiratory function postoperatively by continuous epidural analgesia. The advantage of epidural catheter is that it provides a more stable arterial blood pressure and improved utero placental blood flow in the intraoperative period.?

Patients, especially with hypertensive disorders are susceptible to fluctuations of BP during general anaesthesia and may require use of potent opioids, intravenous anti hypertensives, vasodilators etc to control surge of BP during laryngoscopy and intubation, extubation, and vasopressors following induction to treat hypotension. Whereas during neuraxial anaesthesia the changes in BP are in one direction, that is hypotension. Quick and short acting vasopressors either as intermittent bolus or as infusion along with intravenous fluids are effective in managing hypotension following initiation of neuraxial anaesthesia. However, patients with extremes of blood pressure need monitoring of invasive $\mathrm{BP}$ for continuous monitoring and better 
titration of medications to ensure adequate perfusion pressure. A prospective randomized, multicenter study conducted by Visalyaputra $\mathrm{S}$ et $\mathrm{al}^{8}{ }^{8}$ concluded that SAB was safe for CS in patients with severe preeclampsia. The incidence and magnitude of hypotension following SAB for CS are lesser in patients with severe preeclampsia when compared with healthy parturients ${ }^{9}$ as hypertension is not sympathetically mediated ${ }^{10}$ in preeclampsia.

In our case, the parturient presented with a high BP of 170/100 $\mathrm{mmHg}$ with no imminent symptoms and was therefore started on antihypertensive therapy. As her subsequent BP readings remained around the same in spite of antihypertensive medications, she was taken up for an emergency CS. In the operating room, her BP recorded was $220 / 124 \mathrm{mmHg}$. Since the parturient was not in active labour and there were no imminent symptoms of eclampsia, time constraint for induction of anaesthesia was not a major concern. Therefore, CSE anaesthesia was planned. However, possibility of the patient developing preeclampsia superimposed on gestational hypertension could not be ruled out because repeat blood tests were not obtained.

Cerebrovascular accidents are the uncommon but feared complication contributing to more than $12 \%$ of all maternal deaths. Majority of pregnancy-related strokes occurred either post-partum or at the time of delivery and are ischemic due to arterial occlusion. ${ }^{11}$ Kittner et al. ${ }^{12}$ found an increased risk of ischemic stroke in the postpartum period, the risk of haemorrhagic stroke was increased 2.5fold during pregnancy and 23.8-fold post-partum. Since our patient presented with an unusually high blood pressure and no imminent symptoms, control of hypertension was the primary target so as to prevent occurrence of any cerebro vascular event and thereby protect the health of the mother.

The Royal Australian and New Zealand College of Obstetricians and Gynaecologists (RANZCOG) categorized the urgency for CS as follows:

Category 1: Immediate threat to the life of a woman or foetus.

Category 2: Maternal or foetal compromise, but not immediately life threatening.

Category 3: Needing early delivery but no maternal or foetal compromise. team

Category 4: At a time to suit the woman and the caesarean section

The parturient in our case was grouped into category 2 considering the possible maternal risk due to high BP.

Even though recent meta-analysis and guidelines favour the use of phenylephrine for treating hypotension following $\mathrm{SAB}$ during $\mathrm{CS}^{13,14}$ ephedrine can be safely used for maintaining $\mathrm{BP}$ as it does not adversely affect the uterine blood flow. Berends et al. ${ }^{15}$ have concluded that the use of prophylactic ephedrine is a safe and effective method for prevention and treatment of hypotension after CSE technique. Ephedrine is preferred over phenylephrine in the presence of bradycardia ${ }^{14}$ our patient developed significant hypotension (BP $97 / 56 \mathrm{~mm} \mathrm{Hg}$ ) and a decrease in HR to $68 / \mathrm{min}$ within minutes of administering SAB. This transient drop in BP was treated effectively with Ephedrine, and well tolerated by both the patient and the foetus.

The crux of the management of our patient was quick treatment of dangerously high BP by intravenous Labetalol to reduce it to a more acceptable level with continuous monitoring of invasive BP followed by initiation of CSE. Monitoring invasive arterial BP was very crucial which allowed real-time monitoring, reduction of BP in a controlled manner, quicker administration of vasopressors to treat hypotension, and thereby ensured preservation of both maternal and foetal perfusion. Regional anaesthesia also allowed continuous neurological monitoring. In our opinion, BP should not be the sole criteria to decide which type of anaesthesia, GA or neuraxial, is preferable in a parturient undergoing CS. Other factors like the urgency of CS, overall condition of the mother as well as foetal wellbeing need to be considered before deciding the type of anaesthesia.

\section{Conclusion}

Carefully conducted neuraxial anaesthesia can be useful for caesarean delivery in parturients with severe elevation of blood pressure where general anaesthesia is normally indicated.

\section{Funding details}

None.

\section{Acknowledgments}

None.

\section{Conflicts of interests}

Authors declare that there is no conflict of interest.

\section{References}

1. James PR, Nelson-Piercy C. Management of hypertension before, during, and after pregnancy. Heart. 2004;90(12):1499-1504.

2. Lindheimer MD, Taler SJ, Cunningham FG. Hypertension in pregnancy. J Am Soc Hypertens. 2008;2(6):484-494.

3. Chaudhary S, Salhotra R. Subarachnoid block for caesarean section in severe preeclampsia. J Anaesthesiol Clin Pharmacol. 2011;27(2):169-173.

4. John Jairo PL, Jose RN. Regional versus general anesthesia for cesarean section delivery. Colombian Journal of Anesthesiology. 2012;40(3):203-206.

5. Sibai BM. Diagnosis and management of gestational hypertension and preeclampsia. Obstet Gynecol. 2003;102(1):181-192.

6. Dyer RA, Els I, Farbas J, et al. Prospective, randomized trial comparing general with spinal anesthesia for cesarean delivery in preeclamptic patients with a nonreassuring fetal heart trace. Anesthesiology. 2003;99(3):561-569.

7. Faust JA Jr, Wilson NE. Cesarean section in a patient with unilateral diaphragmatic paralysis, brachial neuritis, and pregnancy-induced hypertension. Anesth Analg. 1992;75(4):622-625.

8. Visalyaputra S, Rodanant O, Somboonviboon W, et al. Spinal versus epidural anesthesia for cesarean delivery in severe preeclampsia:a prospective randomized, multicenter study. Anesth Analg. 2005;101(4):862-868.

9. Aya AG, Mangin R, Vialles N, et al. Patients with severe pre-eclampsia experience less hypotension during spinal anaesthesia for caesarean delivery than healthy parturients:A prospective cohort comparison. Anesth Analg. 2003;97(3):867-872.

10. Moodley J, Jjuuko G, Rout C. Epidural compared with general anaesthesia for caesarean delivery in conscious women with eclampsia. BLOG. 2001;108(4):378-382.

11. Treadwell SD, Thanvi B, Robinson TG. Stroke in pregnancy and the puerperium. Postgrad Med J. 2008;84(991):238-245. 
12. Kittner SJ, Stern BJ, Feeser BR, et al. (1996) Pregnancy and the risk of stroke. N Engl J Med. 1996;335(11):768-774.

13. Moran DH, Perillo M, LaPorta RF, et al.. Phenylephrine in the prevention of hypotension following spinal anesthesia for cesarean delivery. J Clin Anesth. 1991;3(4):301-305.

14. Alday Munoz E, Palacio Abizanda F, De Diego Pdel R, et al. Ephedrine vs. phenylephrine by intravenous bolus and continuous infusion to prevent hypotension secondary to spinal anesthesia during cesarean section:a randomized comparative trial. Rev Esp anestesiol Reanim. 2011;58(7):412-416.

15. Berends N, Teunkens A, Vandermeersch E, et al. A randomized trial comparing low-dose combined spinal-epidural anesthesia and conventional epidural anesthesia for cesarean section in severe preeclampsia. Acta Anaesthesiol Belg. 2005;56(2):155-162. 